Gut, 1973, 14, 847-849

\title{
Amylopectin sulphate (SN-263) coats the gastric mucosal surface
}

\author{
D. S. ZIMMON AND VINCENT MAZZOLA
}

From the Veterans Hospital, New York, New York, and New York University School of Medicine

SUMMARY After the application in vitro of ${ }^{14} \mathrm{C}$ amylopectin sulphate solution to frozen sections of human ulcer-bearing stomach, autoradiography demonstrates localization of amylopectin sulphate to the gastric surface. The affinity of amylopectin sulphate macromolecule for the gastric luminal surface would provide a substrate-bound pepsin inhibitor with a molecular sieve-like action to exclude proteolytic enzymes from the mucosal surface. This protective coating action may mimic the hypothetical role of endogenous gastric mucous substances and account for the action of amylopectin sulphate in human peptic ulcer.

Amylopectin sulphate (SN-263) (Cook, Eich, and Cammarata, 1963) is a synthetic water-soluble macromolecule (molecular weight $60 \times 10^{6}$ ) containing 1.6 sulphate ester groups per glucose unit that has a specific capacity to inhibit enzymatic digestion by pepsin in solution (Cook et al, 1963). Its systematic development was based upon the hypothesis that reduced peptic digestion would promote healing of gastric and duodenal ulcers (Bianchi and Cook, 1964). Controlled clinical trials of SN-263 in man have demonstrated its efficacy in accelerating healing of gastric ulcer (Zimmon, Miller, Cox, and Tesler, 1969) and preventing recurrence of duodenal ulcer (Sun and Ryan, 1970). Although conceived as an enzyme inhibitor, recent investigations suggest an alternative mechanism of action based on substrate binding (Cammarata, Bianchi, and Fago, 1971). To evaluate this possibility we have studied the localization of ${ }^{14} \mathrm{C} \mathrm{SN}-263$ in the human ulcer-bearing stomach.

Immediately after delivery of the distal stomach at the time of gastric resection for peptic ulcer, full-thickness $1 \mathrm{~cm}$ squares of the distal gastric lesser curvature within $9 \mathrm{~cm}$ of the pylorus were cut, orientated, and sectioned frozen at 3 microns in a cryostat. Unfixed frozen sections were mounted on numbered slides and stored at $0^{\circ} \mathrm{C}$. Sections were processed unfixed or after $\mathbf{3 0}$ minutes' fixation in formol saline and five minutes' washing in water (three changes). A neutral $0.75 \%$ solution of ${ }^{14} \mathrm{C}$ SN-263 in water $(0.341 \mu \mathrm{Ci} / \mathrm{mg})$ was pipetted to cover the sections uniformly. Sections were held for

Received for publication 25 July 1973 one hour at $28^{\circ} \mathrm{C}$ in a humidified chamber to allow labelling. Sections were then washed in 10 changes of distilled water over one hour to remove excess ${ }^{14} \mathrm{C} \mathrm{SN}-263$ and stripping film was applied. After exposure (42-90 days), developing, fixing, and washing, the autoradiographs were stained with $0.5 \%$ toluidine blue.

Control slides prepared with unlabelled SN-263 demonstrate only random background exposure. Examination of serial fixed and unfixed section pairs shows a marked reduction of ${ }^{14} \mathrm{C} \mathrm{SN}-263$ on fixed sections. Since unfixed sections are partially autolysed and stain poorly, an attempt was made to apply ${ }^{14} \mathrm{C} \mathrm{SN}-263$ before fixation. Again a marked reduction in autoradiographic grains was noted, indicating displacement of SN-263 by formol fixation. Further attempts to reduce autolysis by the application of ${ }^{14} \mathrm{C} \mathrm{SN}-263$ and washing at $4{ }^{\circ} \mathrm{C}$ also reduced labelling.

Microscopic examination of formol-fixed, fullthickness stomach sections revealed autoradiographic grains restricted to the mucosal surface. No increase in grains above background was observed in the lamina propria or in deeper structures. High-power examination of a gastric pit in longitudinal section (fig 1A) reveals grains centred within the pit and absent in lamina propria. The gastric pit in cross section (fig 1B) demonstrates the central position of grains within the lumen. The lamina propria contains only random background grains and testifies to the absence of non-specific tissue localization. Examination of the gastric mucosal surface (fig 1C) shows autoradiographic grains localized to the luminal mucosal surface. 


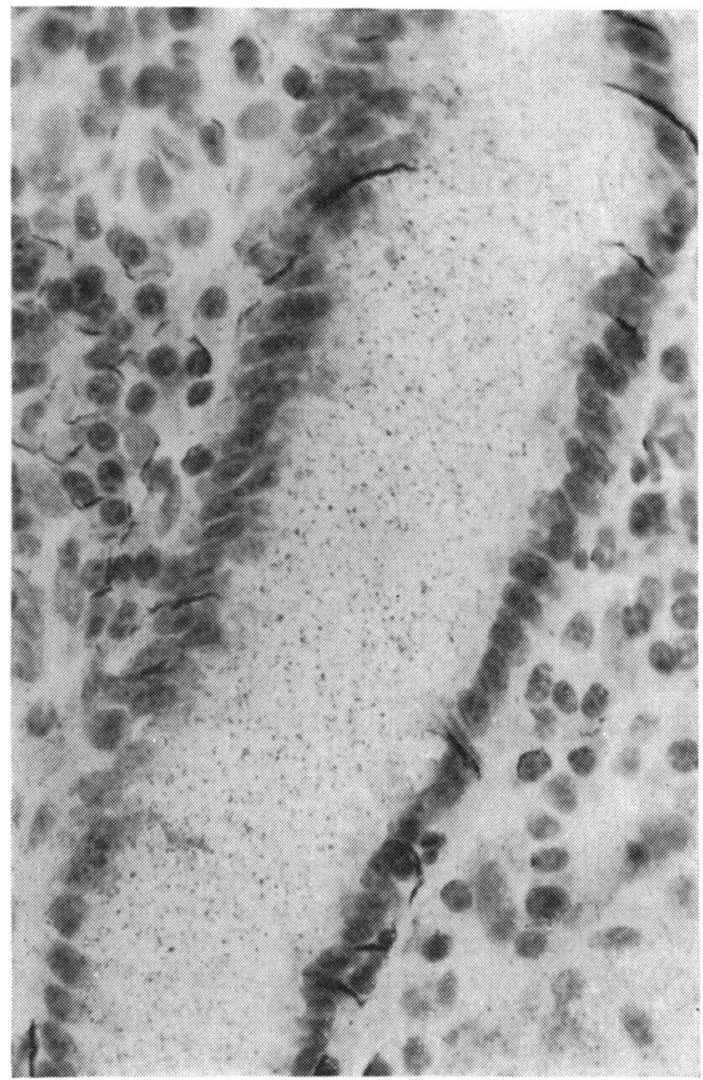

Fig 1A

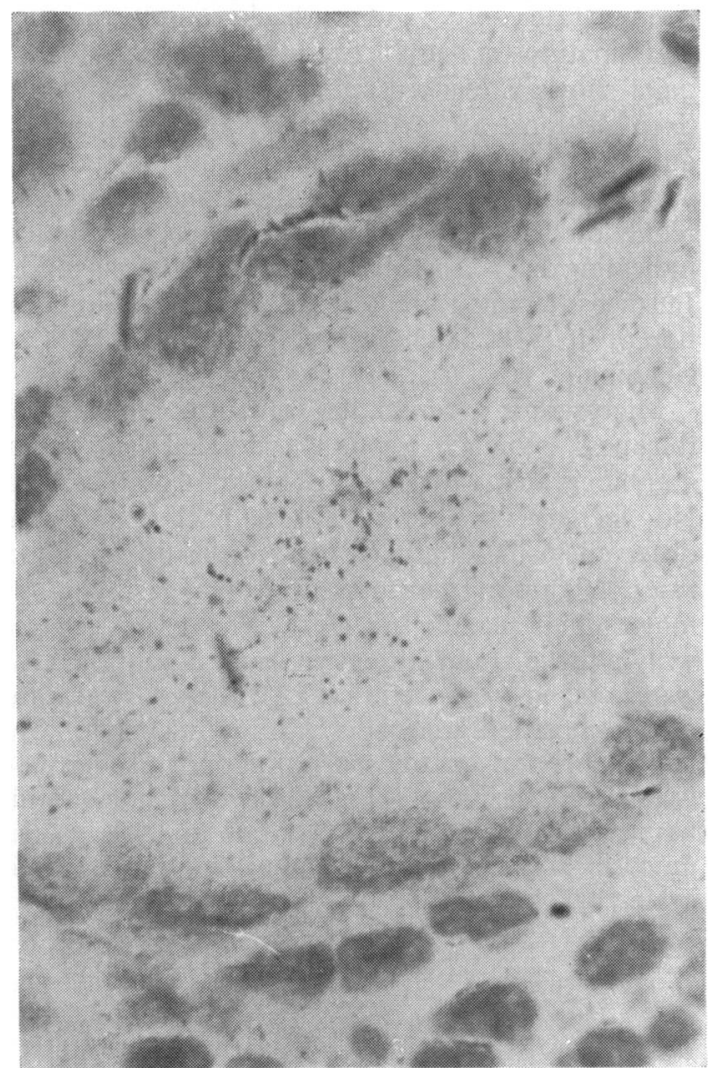

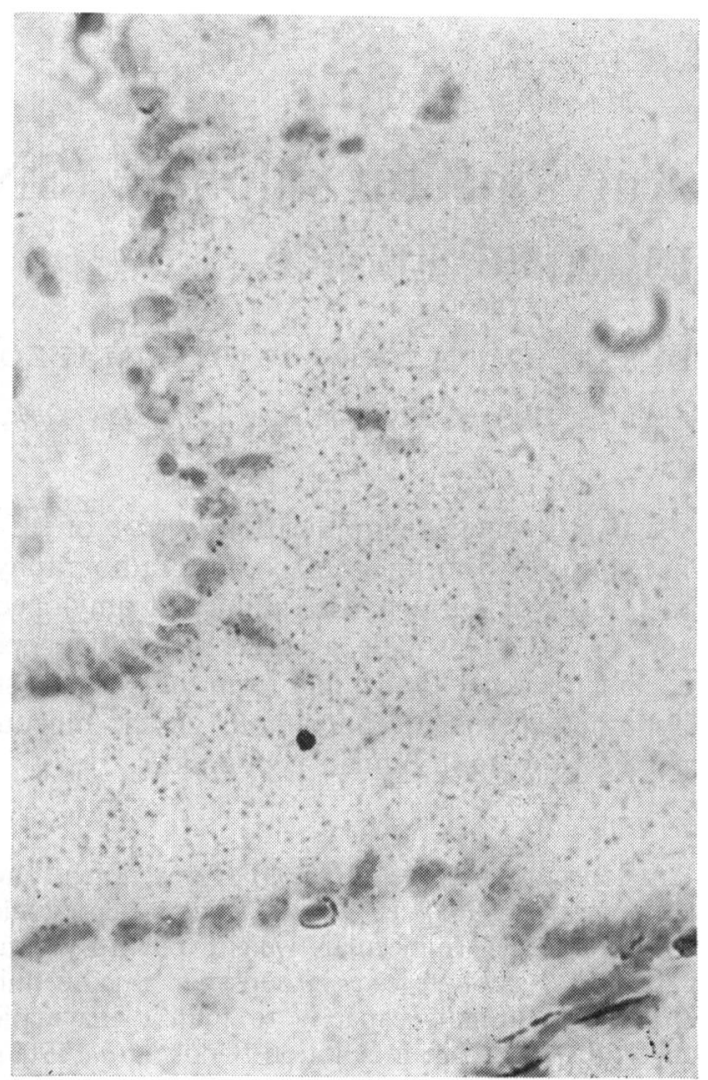

Fig 1C

Fig 1 Autoradiograph of gastric frozen sections toluidine blue stain, fixed.

A Longitudinal section of gland with silver grains indicating ${ }^{14} \mathrm{C} \mathrm{SN}-263$ localized to lumen $(\times 400)$.

B Gland cross section showing central cluster of grains within lumen $(\times 1000)$.

C Gastric mucosal surface at entrance of gastric gland with grains showing luminal mucosal coating by $S N-263(\times 400)$. 
Our results confirm the previous observations of Cammarata et al (1971) indicating an important substrate binding action of $\mathrm{SN}-263$. He showed that (1) in solution substrate bound SN-263 is a more efficient inhibitor of peptic proteolysis than pepsinbound SN-263; (2) SN-263 binds to the surface of heat-coagulated albumin and inhibits peptic proteolysis of the coated solid substrate; (3) a visible methylene blue: SN-263 complex is retained on dog gastric mucosa in vivo for four hours. The latter two observations have been confirmed in man (Zimmon, 1972; Zimmon and Cuy, 1972). This study was designed to confirm the binding of SN-263 to human ulcer-bearing gastric mucosal surface and obviate the criticism that methylene blue: SN-263 complex might not behave identically to SN-263.

The sulphated glycoproteins found in normal gastric juice arise from salivary, bronchial, and oesophageal secretions that contaminate gastric juice as 'adhering sulphated mucus' (Lambert, André, and Berard, 1971). Thus, synthetic sulphated amylopectin behaves in a fashion similar to endogenous sulphated glycoproteins by coating the gastric mucosal surface. A role for endogenous sulphated glycoproteins in protecting gastroduodenal mucosa from peptic ulceration has been postulated on epidemiological grounds (Malhotra, 1970). The binding of ${ }^{14} \mathrm{C} \mathrm{SN}-263$ to the gastric mucosal surface revealed by autoradiography supports a mechanism of pepsin inhibition based upon surface coating. Particularly in the stomach and duodenum where continued secretion and emptying limit the persistence of luminal pepsin inhibitors, mucosal surface coating would prolong the duration of antipeptic action. The sulphated amylopectin macromolecules could form a mucin-like protective molecular sieve excluding proteolytic enzymes but passing smaller molecules. This protective coating action may mimic the hypothetical role of endogenous mucous substances and account for the observed therapeutic action of SN-263 in human peptic ulcer.

This work was supported by the Veterans Administration. ${ }^{14} \mathrm{C} \mathrm{SN-263}$ was kindly supplied by $\mathbf{P}$. S. Cammarata, Ph.D, of G. D. Searle and Company, Skokie, Illinois.

\section{References}

Bianchi, R. G., and Cook, D. L. (1964). Antipeptic and antiulcerogenic properties of a synthetic sulfated polysaccharide ( $\mathrm{SN}-263$ ). Gastroenterology, 47, 409-414.

Cammarata, P. S., Bianchi, R. G., and Fago, F. J. (1971). Mechanism of the antipeptic action of amylopectin sulfate ( $\mathrm{SN}-263)$, an antiulcer, mucin-like agent. Gastroenterology, 61, 850-857.

Cook, D. L., Eich, S., and Cammarata, P. S. (1963). Comparative pharmacology and chemistry of synthetic sulfated polysaccharides. Arch. int. Pharmacodyn., 144, 1-19.

Lambert, R., André, C., and Berard, A. (1971). Origin of the sulfated glycoproteins in human gastric secretions. Digestion, 4, 234. 249.

Malhotra, S. L. (1970). New approaches to the pathogenesis of peptic ulcer based on the protective action of saliva. Amer. J. dig. Dis., 15, 489-496.

Sun, D. C. H., and Ryan, M. L. (1970). A controlled study of the use of propantheline and amylopectin sulfate (SN-263) for recurrences in duodenal ulcer. Gastroenterology, 58, 756-761.

Zimmon, D. S. (1972). Unpublished observations.

Zimmon, D. S., and Cuy, R. (1972). Gastric proteolysis in man and its inhibition by sulfated amylopectin. (Abstr.) Gastroenterology, 62,834.

Zimmon, D.S., Miller, G., Cox, G., and Tesler, M. A. (1969). Specific inhibition of gastric pepsin in the treatment of gastric ulcer. Gastroenterology, 56, 19-23. 